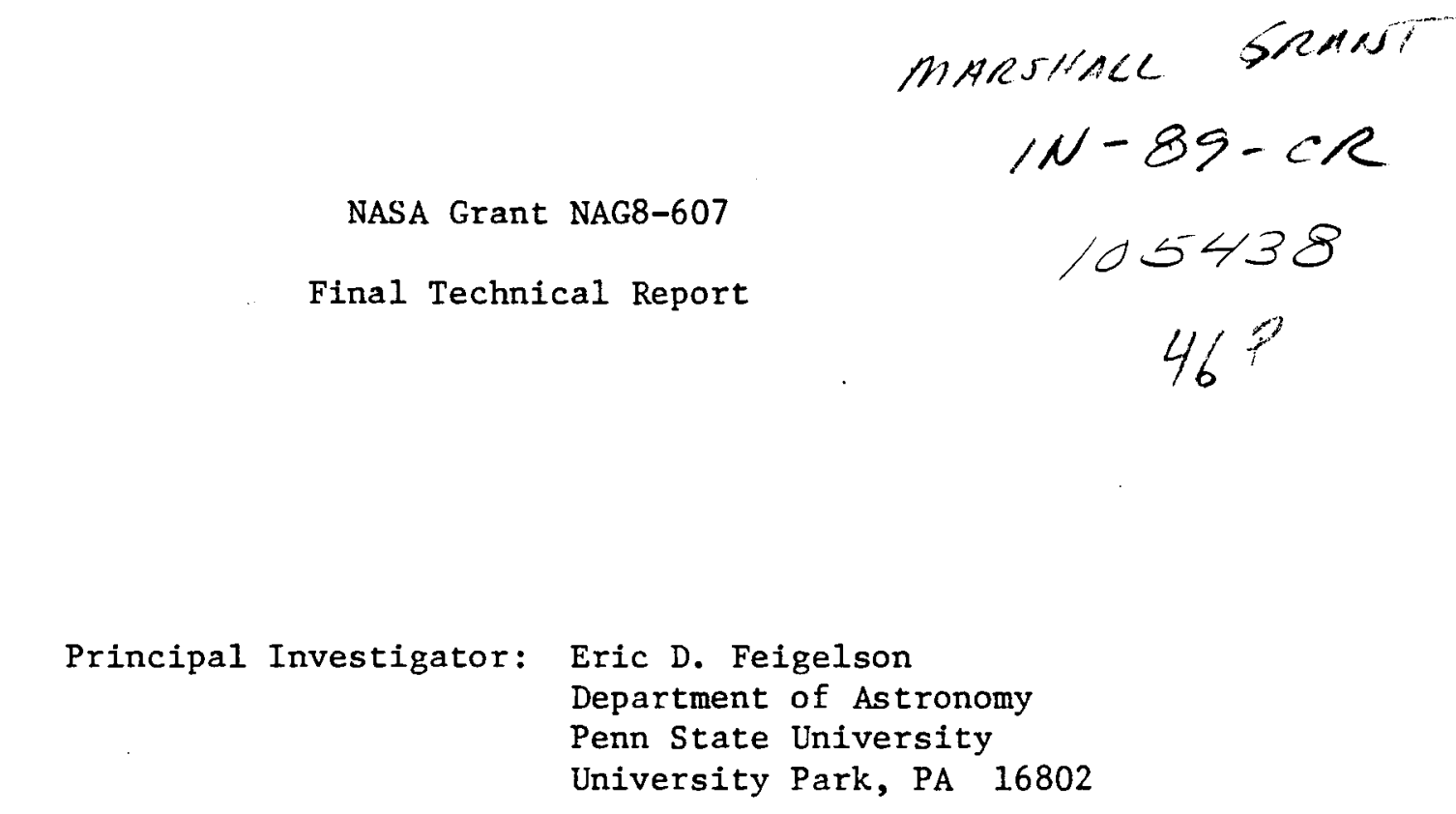

Grant Period: October 1, 1986 to September 30, 1987
Grantee Institution: Pennsylvania State University Office of Sponsored Programs 207 old Main Building University Park, PA 16802

(WASA-CR-181457) SOPT X-RAY OBSERVAT IONS OP PRE-MAIN SEQUENCE STARS IN TRE CHAHAELEOU DARK CLOND (Pennsy lrania State Oniv.) $46 \mathrm{p}$ Avail: NTIS HC $03 / 4$ A01 CSCL O3A 


\title{
Soft X-ray Observations of Pre-Main Sequence Stars in the Chamaeleon Dark Cloud
}

\author{
Eric D. Feigelson ${ }^{1,2}$ and Gerard A. Kriss ${ }^{3}$
}

1) Department of Astronomy

Penn State University

University Park PA 16802

2) NSF Presidential Young Investigator

3) Department of Physics and Astronomy

Johns Hopkins University

Baltimore MD 21218 


\section{Abstract}

Einstein IPC observations of the nearby Chamaeleon I star forming cloud show 22 well-resolved soft $X$-ray sources in a $1^{\circ} \times 2^{\circ}$ region. Twelve are associated with Ho emission line pre-main sequence (PMS) stars, and four with other optically selected PMS stars. Several X-ray sources have two or more PMS stars in their error circles. Optical spectra were obtained at CTIO of possible stellar counterparts of the remaining $X$-ray sources. They reveal 5 probable new cloud members, $\mathrm{K} 7$ MO stars with weak or absent emission lines. These 'naked' X-ray selected PMS stars are similar to those found in the Taurus-Auriga cloud.

The spatial distributions and $H-R$ diagrams of the $X$-ray are optically selected PMS stars in the cloud are very similar. Luminosity functions indicate the Chamaeleon stars are on average -5 times more $X$ ray luminous than Pleiad K-dwarfs. A significant correlation between $L_{x}$ and optical magnitude suggests this trend may continue within the PMS phase of stellar evolution. The relation of increasing X-ray luminosity with decreasing stellar ages is thus extended to stellar ages as young as $10^{6}$ years.

Subject headings: Stars: Evolution; Stars: Pre-Main-Sequence; Stars: X-rays; X-rays: Sources 


\section{Introduction}

Recent progress in X-ray astronomy is leading to major changes in our knowledge of the pre-main sequence (PMS) phase of stellar evolution. PMS stars were traditionally found in optical $\mathrm{H} \alpha$ emission line surveys or in infrared surveys of dark clouds. These techniques gave samples of 'T Tauri' stars around $10^{6}$ years old and various types of younger protostellar objects (see reviews by Cohen 1984, Bertout 1984 and shu et al. 1987). X-ray surveys with the Einstein X-ray Observatory detect some $T$ Tauri stars at levels $-10^{3}$ times those seen in typical main sequence stars, but also reveal large numbers of previously unknown $\mathrm{X}$ ray luminous PMS stars having neither strong emission lines or infrared excesses (see reviews by Feigelson 1984, 1987 and Walter 1987). The strong and variable X-ray emission is best understood as high levels of solar-type magnetic surface activity.

Several nearby regions of star formation have been studied in $\mathrm{X}$ rays to date. The Taurus-Auriga cloud complex (e.g. Feigelson and DeCampli 1981, Walter and Kuhi 1981, Feigelson et al. 1987), $\rho$ Ophiuchi cloud (Montmerle et al. 1983) and Orion nebula (e.g. Ku et al. 1982; Zoonematkermani and Caillault 1987) are the most populous clouds, each with several dozen X-ray emitting PMS stars. Portions of the NGC 2264 , R Cr Austr, and other clouds have also been examined. Optical studies of the $\mathrm{X}$-ray discovered stars in Taurus-Auriga shows most are coeval with and similar to T Tauri stars, except for their lack of circumstellar material (Feigelson and Kriss 1981; Mundt et al. 1983; Walter 1986; Walter et al. 1988). A few, however, lie far from dark clouds and near the ZAMS, indicating they may be 'post-T Tauri' stars. 
We report here $\dot{X}$-ray observations of another nearby star forming region, the Chamaeleon I cloud. In many ways it is well-suited for study: it can be covered more completely than the Taurus-Auriga complex, suffers less obscuration than the $\rho$ Ophiuchi cloud, and is closer than the Orion nebula. Its previously known stellar population consists of $44 \mathrm{H} \alpha$ emitting stars, 7 non-emission line stars with variable star designations, 2 B9.5 stars, a few additional $2 \mu$ infrared sources, and possibly additional IRAS stellar sources (Schwartz 1977; Hyland et al. 1982; Schwartz and Henize 1983; Baud et al. 1984; Jones et al. 1985; Whittet et al. 1987). Seven of these stars illuminate reflection nebulae and three have ejected Herbig-Haro objects. Radio studies of $\mathrm{OH}$ and $\mathrm{H}_{2} \mathrm{CO}$ lines reveal a 'dwarf molecular cloud' with $\mathrm{M}_{\text {gas }} \approx 700-1000 \mathrm{M}_{\mathrm{O}}$ ' possibly connected to Gould's Belt (Toriseva et al. 1985; Toriseva and Mattila 1985).

We find 22 well-resolved X-ray sources, most associated with previously identified PMS stars but a few probably new cloud members. Section II describes the $\mathrm{X}$-ray observations and optical spectroscopy of some candidate stellar counterparts. Section III discusses the stellar identifications. Section IV examines the statistical relations between $\mathrm{X}$-ray and optical properties, including the X-ray luminosity function of PMS stars. The findings are summarized in Section $V$. An Appendix provides detailed information on the $X$-ray emitting stars. We note that the distance to the Chamaeleon cloud has been the subject of considerable debate, with estimates ranging from 115 to $215 \mathrm{pc}$, due to difficulties in obtaining a consistent extinction law for the cloud. We adopt a value of $140 \mathrm{pc}$, based on the study of reddening of background stars around the cloud by Whittet et al. (1987). 
II. Observations and Analysis

(a) X-ray Observations

The Chamaeleon I cloud was observed with the Imaging Propotional Counter (IPC) on board the Einstein X-ray Observatory (HEAO-2 satellite) for a total of 2.5 hours on 23-24 January 1981, about 3 months before the Observatory ceased operations. The instrument is described by Giacconi et al. (1979). Since the cloud is about $2^{\circ}$ in extent and the IPC field of view is about $1^{\circ}$, three overlapping IPC exposures were obtained. They are described in Table 1. The IPC gain was high (ALP = 17) and constant during the observations. A composite contour map of the three fields is shown in Figure 1.

The exposure of the central field, I10349, was subject to an unusual failure of the satellite aspect system. The cause is not known, but may be related to errors in Skymap guidance star positions in the extreme southern sky. The satellite pointing was set by maintaining constant gyroscope readings rather than constant guide star positions in the star trackers. It was thus subject to tidally induced pointing drifts during the observations, and absolute celestial coordinates were not internally determined. Fortunately, the quality of the image seems to be good (e.g., sources do not appear smeared). Absolute alignment of the image was obtained using six X-ray sources seen in the overlapping 10350 image to the south. We estimate this alignment is accurate to $0.3^{\prime}$, which we have added linearly to other sources of positional errors.

$\mathrm{X}$-ray sources were found by passing a $2.4^{\prime} \times 2.4^{\prime}$ cell across each IPC field in three pulse height channel bands, and noting where the 
counts exceeded a specified threshold. Thresholds were set at a fixed level in Rev. 0 processing, and at variable levels to account for vignetting and obscuration by the window support structures in Rev. 1B processing. The Rev. 1B source detection algorithms located 21 distinct sources after eliminating two sources with $S / N \leq 3.0$, which are listed by ' $\mathrm{CHX}$ ' (Chamaeleon $\mathrm{X}$-ray source) number in order of increasing right ascension in Table 2. One additional source (CHX 13) found in Rev. 0 processing that did not exceed the Rev. 1B thresholds is included. CHX $4,7,10,12,14$ and 22 were independently found in overlapping fields; in these cases the data from the central 10349 field is given on a second line. The fourth column of Table 2 gives the estimated 908 error circle radius for each source, including both statistical and systematic uncertainties. The Notes to the Table give cases where the centroids may be systematically displaced due to proximity of the window support structures.

The next three columns give the counts after background subtraction in the $2.4^{\prime}$ cell centered on the centroid position, the local background determined from the MDETECT algorithm, and the maximum signal-to-noise ratio found in Rev. 1B processing. The probability that sources with $S / N \approx 3.0$ are merely statistical fluctuations in the background is not negligible. The eigth column in Table 2 gives the X-ray flux in the $0.2-4 \mathrm{keV}$ band in units of $10^{-13} \mathrm{erg} \mathrm{s} \mathrm{sm}^{-1} \mathrm{~cm}^{-2}$. It is calculated from the count rate corrected for telescope scattering and vignetting, and assuming a conversion of 1 IPC count $s^{-1}=2.5 \times 10^{-11}$ ergs $s^{-1} \mathrm{~cm}^{-2}$. This corresponds to a thermal X-ray spectrum with $\mathrm{kT}-1 \mathrm{keV}$ with foreground column density $N_{H}=3 \times 10^{20} \mathrm{~cm}^{-2}$. Such X-ray spectra, harder than those seen in main sequence stars, is consistent with those meas- 
ured in several Taurus-Auriga PMS stars (Walter and Kuhi 1984) and with our measured hardness ratios. Assuming a cloud distance of $140 \mathrm{pc}, 1.0$ $\times 10^{-13}$ ergs $s^{-1} \mathrm{~cm}^{-2}$ corresponds to $2.4 \times 10^{29}$ ergs $s^{-1}$.

We now consider properties of the X-ray sources beyond their location and strength. Although the sources were generally too faint to perform quantitative analysis of any possible structure, several sources in the cloud appear possibly multiple or extended. $\operatorname{CHX} 9,11,15$ and 16 show both elongated contours and broadened radial profiles, while CHX 19 appears slightly elongated on the contour map alone. Details are given in the Notes to Table 2 . This result must be considered tentative given the absence of definite quantitative tests.

The $\mathrm{X}$-ray sources are also too faint to make reliable least squares fits to their spectra. However, the hardness ratio defined by $[\operatorname{Cts}(0.8-3.5 \mathrm{keV})-\operatorname{Cts}(0.2-0.8 \mathrm{keV})] /[\operatorname{Cts}(0.8-3.5 \mathrm{keV})+\operatorname{Cts}(0.2-0.8$ $\mathrm{keV}$ ] can be measured. It is typically 0.2 to 0.5 for the fainter sources, and appears to be consistently higher at 0.4 to 0.8 for the stronger sources. This trend is opposite to that expected if the stronger sources are less absorbed than the fainter sources, and suggests an intrinsic flux-hardness correlation. Note that the Chamaeleon observations may be more sensitive to energies in the $1-4 \mathrm{keV}$ range than other observations of PMS stars because the IPC detector gain was abnormally high.

We examined each source for possible temporal variations within each exposure without success. However, variability may be present in sources between the 10350 exposure and the 10349 exposure 6 hours later. Five sources ( $\operatorname{CHX} 4,7,10,12$, and 22) appear a factor of $1.1-1.5$ times stronger in the later image, while $\mathrm{CHX} 14$ appears 2.2 times 
weaker. It is difficult to evaluate the reliability of these differences, as sources suffer different amounts of vignetting and (possibly) obscuration by the support structures. For example, CHX 14 in 10349 lies $3^{\prime}$ away from two support structures; this should not have caused a factor of two reduction in flux, but the possibility can not be rigorously excluded. We conclude that variability on a timescale of hours may, but not definitely, have occurred in some sources.

\section{(b) CTIO Observations}

Figure 2 presents finding charts for all the $\mathrm{X}$-ray sources listed in Table 1. The cross hair is positioned at the best fit location of the $\mathrm{X}$-ray source, and the circle is at the 908 confidence radius. Many of the X-ray sources correspond to well-studied emission line PMS stars. The fields of nine of the brightest $X$-ray sources without well-studied counterparts were searched spectroscopically with the $4 \mathrm{~m}$ telescope at the Cerro Tololo Interamerical Observatory on the night of 11 December 1981. The RC Spectrograph and the UV SIT Vidicon detector were used for the observations. Grating \#400, 158 lines/mm blazed at $8000 \mathrm{~A}$, gave spectral coverage from 4000 to $7000 \mathrm{~A}$ and a spectral resolution of $-12 \mathrm{~A}$ with a $300 \mu(-2$ arc sec $)$ slit. The observations were performed during the moonlit portion of a night devoted primarily to the identification of faint, X-ray selected quasars. Four white dwarfs were observed as flux standards throughout the night.

The long slit spectra were rectified using the twodspec package withing the Interactive Reduction and Analysis Facility (IRAF) on a VAX $11 / 750$ at The Johns Hopkins University. The stellar spectra were then 
extracted and sky subtracted from the rectified images using the sumextract routine. Variance weighting was used to give the optical signal to noise ratio in the extracted spectra. Flux calibration was then done in the onedspec package. The sky was clear and the seeing was good; the four white dwarf standards yielded a sensitivity function with a root mean square (RMS) scatter of less than 108 in each calibrated bandpass from 4000 to $7000 \mathrm{~A}$. A montage of all the flux-calibrated spectra is shown in Figure 3. The flux scales for the stars associated with CHX 12 should be considered as relative flux only since these two stars were barely resolved along the slit during a single integration. The extractions of these two spectra were optimized to give the best separation between the spectra rather than preserving the absolute flux level.

Spectral types for each of the observed stars were determined by visual comparison with the spectral catalogue of. Prichet and van den Bergh (1977). We estimate our spectral types to be accurate to within one spectral sub-class (1 sigma) except for $G$ stars which we feel are uncertain to 2 spectral sub-classes. Broad band $B$ and $V$ magnitudes were determined by multiplying the spectra with the bandpasses as given by Allen (1973), integrating to get the total flux, and converting this to a magnitude as prescribed by Allen (1973). R magnitudes were determined by measuring the continuum flux at $7000 \mathrm{~A}$ and converting to a magnitude assuming that $R=0$ corresponds to $1.74 \times 10^{-9}$ ergs $\mathrm{cm}^{-2} \mathrm{~A}^{-1}$. The $\mathrm{R}, \mathrm{B}$, and $V$ magnitudes each have an rms error of $\sim 0.13$ magnitudes based on comparisons with measured values for the white dwarf standards and the star associated with $\mathrm{CHX} 7$. The colors $B-V$ and $V-R$ have slightly smaller dispersions of 0.11 magnitudes. Assuming that all of the stars are 
luminosity class V, we estimated the color excess E(B-V). Errors in $\mathrm{E}(\mathrm{B}-\mathrm{V})$ are assumed to be the sum in quadrature of the error in color and the uncertainty in $B-V$ corresponding to the error in spectral type.

Each stellar spectrum was searched for Ha emission or absorption. Emission and absorption line fluxes and equivalent widths were determined by visually setting continuum points to either side of the feature and integrating between these points. An estimate of the error was made by measuring the rms of the continuum to either side of $\mathrm{H} \alpha$. Since the Ha features are unresolved, the $1 \sigma$ error in the equivalent width is approximately the resolution of 12 A divided by the signal to noise ratio of the continuum. This error in equivalent width was propagated through to the error in the line flux. All these measured quantities are listed in Table 3. 
III. Stellar Counterparts to the $\mathrm{X}$-ray Sources

The likely stellar counterpart(s) for each X-ray source was located by inspection of the finding charts, and by use of the SIMBAD Bibliographic Database at the Stellar Data Center in Strasbourg (Heck and Warren 1986). The latter provided all bibliographic references to objects within each IPC error circle. The detailed characteristics of each possible counterpart, including both previously published data and our spectra discussed in the section above, are presented in the Appendix. We summarize the results here.

of the $22 \mathrm{CHX} X$-ray error circles, 16 contain previously identified PMS stars, 5 contain probable X-ray discovered PMS stars, and one (CHX 5) has no obvious stellar counterpart. Though somewhat subjective, the counterparts might be classified as follows. Five to seven sources ( $\mathrm{CHX}$ $1,16,17,18,19,12$ if the source is VW Cha, 15 if the source is WY Cha) are associated with 'classical T Tauri' stars. These possess most or all of the following properties: moderate to strong $\mathrm{H \alpha}$ emission, other optical emission lines, 'veiling' of the spectrum in the blue, ultraviolet (UBV) excess, infrared (JHKL) excess, and photometric variability. Five to seven ( $\mathrm{CHX} 3,4,9,14,20,12$ if the source is $\mathrm{Sz} 23$, 15 if the source is $\mathrm{Sz} 33$ ) are associated with weak or 'naked' $\mathrm{T}$ Tauri stars. These were discovered in $\mathrm{H \alpha}$ objective prism surveys, but have weak to moderate $\mathrm{H} \alpha$ strengths, no other emission lines, and little or no color excesses in the ultraviolet or infrared. Two X-ray sources (CHX $2,8)$ are associated with stars identified by virtue of their photometric variability, and never appeared in emission line surveys. Two sources ( $\mathrm{CHX} 7,22$ ) are $G$ stars illuminating reflection nebulae, with no 
emission lines or photometric variability.

There are two catalogued stars that may be associated with the possible extended structures near $\mathrm{CHX} 9,11,15,16$, and 19 . The Ho star HM 16 - Sz 22 lies 2.1' southeast of HM 13, the proposed identification of $\mathrm{CHX} 9$, and may cause the extended feature east of the centroid. Similarly, the faint Ha star HM 23 = Sz 32 lies 1.1' southwest of WW Cha,

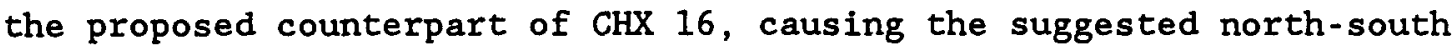
elongation.

Five sources ( $\operatorname{CHX} 6,10,12,13,21$ ) are probable new PMS stars discovered by virtue of their X-ray emission. The error circle of CHX 6 contains an unreddened V $=13 \mathrm{~K} 7$ star and a reddened V $=14$ M0 star, neither of which have emission lines. $\mathrm{CHX} 10$ and 21 each contain a V = $13 \mathrm{~K} 7 \mathrm{star}$; the fainter $\mathrm{F} 3$ star in the $\mathrm{CHX} 21$ error circle is undoubtably a background star. The CHX 10 star is superposed on the densest part of the molecular cloud (Toriseva et al. 1985). CHX 13 is a highly reddened MO star with a weak Ha line. CHX 12 is a confusing case. It was previously reported as a probable cloud member due to an infrared

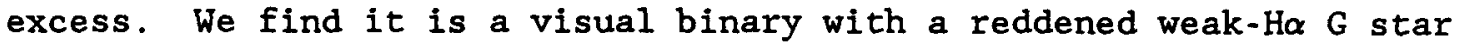
and a less reddened $K$ star. The infrared excess is probably due to the binarity, and we suspect both stars are probable cloud members. It is not clear which is the X-ray emitter.

The one source, $\operatorname{CHX~5,~that~has~no~obvious~stellar~counterpart,~}$ either on the finding chart or in earlier catalogs of cloud members, is probably not a real $\mathrm{X}$-ray source at all. It lies near the edge of field 10350 and just barely triggered the MDETECT source detection algorithm, which can be inaccurate at field edges.

Finally we mention two other types of PMS objects in the cloud. The 
Herbig-Haro objects $\mathrm{HH} 48$ and 49-50 (see Schwartz 1977) were not seen with a flux limit of $2.6 \times 10^{-13}$ ergs $s^{-1} \mathrm{~cm}^{-2}$. H-H 51 coincided with a window support structure and therefore was not observed. The two ZAMS B9.5 stars HD 97300 and 97048 , the latter of which has been extensively studied for its unusual near-infrared emission features, are less clear. HD 97300 lies in a confusing region with a number of embedded PMS objects (Jones et al. 1985) and possibly extended X-ray emission around CHX 16; however, there is no indication of $\mathrm{X}$-rays from the hot star itself. HD 97048, on the other hand, may be associated with the faint eastern extension of $\mathrm{CHX} 9$. If so, its flux would be $-1 \times 10^{-13}$ erg $s^{-1}$ $\mathrm{cm}^{-2}$. Note that several B8 stars in other star formation regions have been detected in Einstein images (e.g. Feigelson and DeCampli 1981; Walter and Kuhi 1981). 
IV Comparison of X-ray and Optical Properties.

Figure 4 shows a diagram of the Chamaeleon cloud with the $\mathrm{X}$-ray sources, optically Ho stars and other PMS objects superposed on an outline of the diffuse obscuring material. The spatial distribution of the $\mathrm{X}$-ray emitting stars is quite similar to that of the Ho stars. Some lie directly in front or within the dark cloud, while others lie at the periphery. The $\mathrm{X}$-ray stars follow the basic clustering pattern seen with other cloud members, showing a clump of PMS stars in the northern part around HD 97300, a second clump in the southern part around HD 97048, and few between or below these groupings. In particular, there was considerable opportunity to pick up a distant or 'post-T Tauri' population of $\mathrm{X}$-ray emitters south of $\delta=-77.5^{\circ}$, which do not appear to be present.

Figure 5 shows a crude Hertzsprung-Russell diagram for the X-ray and $\mathrm{H} \alpha$ stars with measured spectral types. Locations along the ordinate are very uncertain due to possibly large bolometric or reddening corrections. Nonetheless, one can see that the $\mathrm{X}$-ray and $\mathrm{H \alpha}$ populations occupy approximately similar regions of the H-R diagram. A notable difference in the populations is the high fractions of $G$ stars among the $\mathrm{X}$-ray stars, some of which have no $\mathrm{H \alpha}$ emission. These include $\mathrm{CHX} 7=$ Ced 110, $\operatorname{CHX} 9=\operatorname{HM} 13, \operatorname{CHX} 12 \mathrm{~b}, \operatorname{CHX} 19=\mathrm{HM} 30$, and $\operatorname{CHX} 22=\mathrm{HM}$ Anon. They are reminiscent of two PMS $G$ stars in the Taurus-Auriga cloud, SU Aur (Feigelson and DeCampli 1981) and HD 283572 (Walter et al. 1987). They are unusually $X$-ray luminous, rapidly rotating stars with very high levels of magnetic surface activity, and probably have higher masses (M $\sim 2 M_{O}$ ) than later-type PMS stars. Ced 110, in particular, is notable 
for its high X-ray luminosity of several $\times 10^{30}$ ergs $s^{-1}$ and hard $X$-ray spectrum, similar to HD 283572.

The Chamaeleon cloud is susceptible to some quantitative statistical analysis that is not readily performed in other star formation clouds. Some clouds are only partly covered with Einstein IPC images (e.g., Taurus-Auriga), others are too obscured or confused to have good Ho survey coverage (e.g. Orion), while others have good coverage but little overlap between $\mathrm{X}$-ray sources and well-studied $\mathrm{T}$ Tauri stars (e.g. the $\rho$ Ophiuchi cloud).

The statistical analysis proceeds in three stages. First, a welldefined sample of $\mathrm{H \alpha}$ stars that were observed (though not necessarily detected) in the three IPC images is constructed. This is complicated by the ambiguity of stellar counterparts discussed in Section III. Second, the X-ray luminosity function for the Ha sample is calculated. Since both detections and upper limits are present, 'survival analysis' statistical methods appropiate for 'censored data' must be used. These have been developed by statisticians, and are described by Avni et al. (1980), Schmitt (1985), Feigelson and Nelson (1985), and Isobe, Feigelson and Nelson (1986). Third, correlations between X-ray emission and various optical properties of the stars can be sought using bivariate survival analysis methods.

We consider two statistical samples: the sample of X-ray selected PMS stars defined in Table 2, and a sample of optically (i.e. Ho emission) selected stars that were observed, though not necessarily detected, in the IPC images. Constructing the latter sample is complicated by the ambiguity of stellar counterparts discussed in Section III. We start with the sample of $45 \mathrm{H \alpha}$ emission-line stars in the 
Chamaeleon I cloud found with the CTIO Curtis Schmidt telescope by Schwartz (1977). Five of these stars (Sz 1, 4, 8, 18 and 45) lay outside the IPC fields, and three (Sz 17, 28 and 31) lay very close to the obscuring window support structures; these are considered unobserved. Nine Schwartz stars are unambiguously detected $(\operatorname{CHX} 1,3,4,9,14,16$, 17, 18, and 20). Three CHX sources have two Schwartz stars in their error circles (CHX 11, 15 and 19), and three Schwartz stars may or may not be responsible for marginally resolved X-ray emission (Sz 22 near $\mathrm{CHX} 9$ and $\mathrm{Sz} 32$ near $\mathrm{CHX} 16$ ). We chose to consider these nine stars to be 'unobserved'. This omission will bias downwards the inferred X-ray luminosity function, as some of them undoubtably are X-ray emitters, but will avoid erroneous conclusions resulting from incorrect assignments of $\mathrm{X}$-ray emissivity. Finally, we exclude one additional star with proper motion inconsistent with cloud membership ( $\mathrm{Sz} 27$; see Whittet et al. 1987). The sample of $\mathrm{Ha}$ stars for which reliable $\mathrm{X}$-ray observations exist now consists of 27 stars, of which 9 are detected. These are listed in Table 4. For the detected stars, luminosities are calculated from the fluxes in Table 2 assuming a cloud distance of 140 pc. When detections in overlapping fields are present, the average flux is used. For the 18 undetected stars, upper limits are derived from the appropriate IPC field's on-axis sensitivity limit with an off-axis vignetting correction.

For the second stage, the integral $X$-ray luminosity function for optically-selected Chamaeleon stars and its variance is calculated using the Kaplan-Meier product-limit estimator, which is the unique maximumlikelihood estimator of the underlying luminosity distribution (Feigelson and Nelson 1985). Figure 6 shows the result. We also show the 
luminosity function of X-ray selected Chamaeleon stars, which is a simple plot of the $22 \mathrm{CHX}$ sources without any complicated sample definition or mathematical treatment. The formal mean $\log \mathrm{X}$-ray luminosities $<\log$ $L_{x}>$ is $29.82 \pm 0.06$ and $30.04 \pm 0.06$ ergs $s^{-1}$ for the optically- and $X-$ ray-selected samples respectively. The two luminosity functions are shown in Figure 6.

Luminosity functions of Pleiades, Hyades, and field stars derived in a similar fashion by Micela et al. (1987) are plotted for comparison. The mean luminosity of the Ha Chamaeleon sample is factors of 2 and 5 above the mean luminosities of Pleiad and Hyad G stars, and 3 and 16 times the mean luminosities of Pleiad and Hyad $\mathrm{K}$ stars respectively. We recommend caution, however, in using this result quantitatively. Uncertainties in the Chamaeleon mean luminosities include the fact that the shape of the Kaplan-Meier estimator is unknown below the 758 level, the high- $L_{\mathbf{x}}$ end was artificially lowered by the exclusion of ambiguous sources, and the distance is uncertain by as much as \pm 508 . We nonetheless find the trend shown in Figure 6 to be persuasive: the X-ray luminosity function of late-type stars decreases monotonically with stellar age between $10^{9}$ and $10^{6}$ years, with both $X$-ray- and optically-selected PMS stars several times more luminous than open cluster stars.

For the third stage, correlations between the X-ray luminosities and a variety of other properties of the Chamaeleon PMS stars are made. Here, we restrict the analysis to the optically-selected sample in Table 4 to avoid ambiguities in the source identifications. The existence of a correlation was tested using Cox regression, a test commonly used in biostatistics (Isobe, Feigelson and Nelson 1986). The following covariates were examined: red magnitudes, $\mathrm{H} \alpha$ and $\mathrm{Ca}$ II emission line inten- 
sity classes from Schwartz (1977); V magnitudes, spectral types, equivalent widths of $\mathrm{H} \alpha$, He I, O I lines, and Balmer decrements from Appenzeller et al. (1983); V magnitudes and $\mathrm{H} \alpha$ intensity classes from Henize and Mendoze (1973); corrected J-H and H-K color indices from Hyland et al. (1982); $\mathrm{K}$ magnitudes and $\mathrm{J}-\mathrm{H}$ colors from Glass (1979); Ho equivalent widths from Rydgren (1980); and $\mathrm{V}$ magnitudes, $\mathrm{B}-\mathrm{V}$ and $\mathrm{U}-\mathrm{B}$ color indices from Grasdalen et al. (1975). Redundant tests were made because many of the stars exhibit broad-band and spectroscopic variability.

The results of these correlations proved to be very clear. No relation between $\log L_{\mathbf{x}}$ and any spectral characteristic - spectral type, color index, or emission line strength -- was found. The Cox probabilities that the variables are uncorrelated were all found to exceed 158. However, X-ray luminosity was consistently found to be correlated with optical magnitudes. The Cox probabilities ranged from 2.38 to 0.058 significance levels for the $V$ and $R$ band magnitudes, depending on which optical data set was used. Table 4 and Figure 7 present the data using the most complete optical data set (Schwartz 1977). The figure shows the significant correlation between between $\mathrm{X}$-ray and optical brightness, and the uncorrelated scatter plot between X-ray and Ha brightness. We note that an anti-correlation between $L_{x}$ and $H \alpha$ intensity was reported by Walter and Kuhi (1981) in T Tauri stars, and was interpreted as a 'smothering' of X-rays from the stellar surface by the circumstellar envelope. We do not find this anti-correlation in Chamaeleon stars, nor was it found in $\rho$ Ophiuchi PMS stars by Montmerle et al. (1983). 
Twenty-two we1l-resolved X-ray sources are found in three overlapping IPC images of the Chamaeleon I cloud. All but one have at least one probable late-type PMS star in their error circles. (The remaining source is probably a fluctuation in the detector background.) Assuming a cloud distance of $140 \mathrm{pc}$, their soft X-ray luminosities range between $4 \times 10^{29}$ and $4 \times 10^{30}$ ergs $s^{-1}$. Several fainter sources just barely resolved (within $-2^{\prime}$ ) of the definite sources may also be present. The $\mathrm{X}$-ray spectra are quite hard and variability between two exposures separated by 6 hours may, but not definitely, have occurred in a few sources.

of the 21 stellar CHX sources, 16 are probably associated with previously catalogued PMS stars in the cloud. Five to 7 are 'classical T Tauri' stars, 7 - 9 'naked T Tauri' stars, and two G stars illuminating reflection nebulae. The remaining $5 \mathrm{CHX}$ stars are associated with new probable PMS stars identified by our CTIO spectra. Four are K7 or MO stars with different amounts of extinction, and one is a K3/G5e visual binary. Statistical analyses give X-ray luminosity functions of welldefined samples of X-ray-and optically-selected PMS stars in the cloud. They also show X-ray and optical luminosities are significantly correlated, though X-ray emission is statistically independent of color excesses, emission line strengths or spectral types.

In most respects, these results support and extend earlier X-ray findings in the Taurus-Auriga, $\rho$ Ophiuchi and other nearby star forming clouds. Soft X-ray surveys with sensitivities around $3 \times 10^{29} \mathrm{erg} \mathrm{s}$ consistently detect $-1 / 3$ of the $\mathrm{H} \alpha$-emitting stars and reveal previously 
unremarked late-type PMS stars with few of the optical, infrared or ultraviolet anomalies characteristic of $T$ Tauri stars. While we report here 5 probable new cloud members, experience with the other clouds predicts that X-ray observations with more spatial and temporal coverage would uncover more new PMS stars. The Chamaeleon results also confirm that PMS X-ray emission has no important relation to circumstellar material; it is neither produced by nor absorbed in the $\mathrm{H \alpha}$ or infrared emitting disks or envelopes. We further confirm that PMS X-ray spectra are hard, extending to several $\mathrm{keV}$ in the more $\mathrm{X}$-ray luminous Chamaeleon stars. All these lines of evidence support the model (e.g. Feigelson 1984 and references therein) wherein PMS X-ray emission is a product of enhanced solar-type magnetic activity, consistent with other (see Skumanich 1972) indications of higher surface activity in younger latetype stars.

The finding with perhaps the broadest implications is the first construction of PMS X-ray luminosity functions (Figure 6). Though there are some ambiguities regarding precisely how many independent $\mathrm{X}$-ray sources are present and which star is responsible for each, there is comfort in the fact that the luminosity functions for both X-ray- and $\mathrm{H} \alpha$-selected samples are quite similar. The mean $\mathrm{X}$-ray Iuminosity, <log $L_{X}>10^{30.0}$ assuming a cloud distance of $140 \mathrm{pc}$, is -5 times higher that Pleiad $\mathrm{K}$ stars and -100 times higher than main sequence field stars. The trend of $\mathrm{X}$-ray luminosity increasing with stellar youth found by Micela et al. (1987 and references therein) is extended at least an order of magnitude in age down to $-10^{6}$ years. The temporal decay of stellar X-ray emission seems distinctly different from the temporal behavior of chromospheric activity. Walter et al. (1988) show 
that $\mathrm{Ca}$ II and $\mathrm{Mg}$ II line fluxes (once classical $\mathrm{T}$ Tauri stars with confusing circumstellar material are excluded) are relatively constant for stars between $10^{6}$ and $10^{9}$ years. This may indicate a difference in the decay rates of flares (or possibly coronal activity) responsible for the $\mathrm{X}$-rays and chromospheric activity.

Though not fully warranted by the data, we can attempt to construct an analytic formulation of the evolution of X-ray emission in late-type stars, analogous to the Skumanich (1972) relations. Figure 7a clearly shows $L_{x}$ decreases with $m_{r}$. The effect can crudely be modeled by a linear trend where $\left\langle\log L_{x}>\approx 30.5\right.$ at $m_{r} \approx 11$ and $\left\langle\log L_{x}>\approx 29.5\right.$ at $m_{r}$ $\simeq 16$. Comparison of the Hertzsprung-Russell diagram in Figure 5 to the PMS isochrones of Cohen and Kuhi (1979) suggests the $m_{r}-11$ Chamaeleon stars are $-1 \times 10^{6}$ years old and the $m_{r}=16$ stars are $-2 \times 10^{7}$ years old. We then add the mean X-ray luminosities of Micela et al. (1988), assuming the age of the Pleiades is $-5 \times 10^{7}$ years, the Hyades is $-6 \times$ $10^{8}$, young disk stars is $-2 \times 10^{9}$ years and old disk stars is $-1.5 \mathrm{x}$ $10^{10}$ years. The result is a skumanich-type relation where $\left\langle\log L_{x}>\approx\right.$ -0.6 to $-0.7 \log t$. The relation would apply to stars between $10^{6}$ and $10^{10}$ years old, and is normalized at $\left\langle\log L_{X}>\approx 29.5\right.$ and $\log t-9.0$. We consider this a speculative but interesting implication of the Chamaeleon data.

Acknowledgements. We would like to thank the staff of the Einstein Observatory for their efforts at reprocessing field 10349, and W. Warren and A. Heck for providing access to SIMBAD. This work was principally supported by NASA grant NAG8-607; additional support was provided by 
NSF grant AST 83-51447. 
Appendix

Notes on Individual X-ray Emitting Stars

Common designations are: S - Hoffmeister 1963; HRC - Herbig and Rao 1972 catalog; HM - Henize and Mendoza 1973; Sz - Schwartz 1977; T Whittet et al. 1987; IRAS = IRAS Point Source Catalog, taken from Whittet et al. 1987. Principal references, obtained from the SIMBAD Bibliographic Database, are given for each star.

$\mathrm{CHX}$ 1. SY Cha $=\mathrm{HM} 2-\mathrm{Sz} 3-\mathrm{S} 6321=\mathrm{T} 4=\mathrm{IRAS} 10552-7655$. This T Tauri star has moderately strong Balmer lines, weak metal lines, strong ultraviolet veiling, little infrared excess, and can exhibit inverse-P Cygni line profiles. Its spectral type is variously reported as $\mathrm{K} 2$ and $\mathrm{MO}$ :. Most times it shows small amplitude irregular variability around $14<\mathrm{V}<13$. However, for several years in the early 1970's it produced an extraordinarily luminous and blue $(\Delta B=1.6)$ extra component which varied with a strict 6.1 day periodicity. It probably was a rotationally modulated 'starspot', though it is not clear whether it was due to magnetic activity or accretion. [Grasdalen et al. 1975; Appenzeller 1977; Mundt and Bastian 1980; Appenzeller et al. 1983; Schaeffer 1983; Whittet et al. 1987]

CHX 2. SZ Cha = S 6323 - Glass V - T6 - IRAS 10570-7701. This is a PMS star discovered by virtue of its optical variability rather than its H $\alpha$ emission line. It is a KO star lying -2 magnitudes above the ZAMS with $\mathrm{V}$ typically seen between 11.7 and 12.9 . It sometimes exhibits weak $\mathrm{H} \alpha$ emission, E.W. $-7 \mathrm{~A}$ on one occasion, $\mathrm{Ca}$ II $\mathrm{H}$ and $\mathrm{K}$ emission cores, 
and a moderate infrared excess. Broad-band colors vary considerably, with $1.0<\mathrm{B}-\mathrm{V}<1.5$ and $0.4<\mathrm{U}-\mathrm{B}<1.1$. [Grasdalen et al. 1975; Glass 1979; Rydgren 1980; Whittet et al. 1987]

CHX 3. LkHa 332-20 - HRC 244 - HM 4 - Sz 6 - T 8 = IRAS 105787645. One of the visually brightest stars in the cloud with $\mathrm{V}=11.3$, spectral type G8-K2, weak emission lines and little photometric variability. It is a moderately rapid rotator, with spectroscopically measured $v \sin (i)=35 \pm 5 \mathrm{~km} \mathrm{~s} \mathrm{~s}^{-1}$ and photometrically measured $\mathrm{P}=2.3$ days. [Henize and Mendoza 1973; Rydgren 1980; Appenzeller et al. 1983; Bouvier et al. 1986]

CHX 4. HM 7 - Sz $9=\mathrm{T} 11$ - IRAS 11011-7717. Similar to LkH $\alpha$ 332-20 above, this $V \simeq 11.6$ star has a fuller series of Balmer and iron emission lines. The spectral type is variously classified as $\mathrm{K} 2, \mathrm{~K} 5$ and M0. [Henize and Mendoza 1973; Appenzeller 1977; Rydgren 1980; Appenzeller et a1. 1983]

CHX 6. Neither of the two $V=12-13$ stars in this error circle are previously catalogued. Our CTIO spectra show a heavily reddened MO and an unreddened KO star, both without emission lines. We predict that higher resolution spectroscopy will show high lithium and/or $\mathrm{Ca}$ II emission cores in one of these stars. [This paper, Table 3]

CHX 7. Glass F = star exciting reflection nebula Ced $110=\mathrm{T} 21=$ IRAS 11048-7606. This is a G0-G5 star with no emission lines. The absorption features appear 'diffuse or veiled' (Rydgren 1980 and our Figure 3), caused perhaps by rapid rotation. It is the most luminous star in the cloud in X-rays, and one of the brightest optically as well. 
[Glass 1979; Rydgren 1980; Schwartz and Henize 1986; this paper, Table 3]

CHX 8. UX Cha $=\mathrm{S} 6337=\mathrm{T} 22$. Like $\mathrm{CHX} 2$, this is a star identified by its optical variability but with no emission lines. It is rather faint $(15.2<V<16.2)$ and red $(B-V \simeq 2.0)$, suggesting it is an $M$ star. No spectrum is available. [Grasdalen et al. 1975; Rydgren 1980]

CHX 9. CoD $-76^{\circ} 486=\mathrm{CPD}-76^{\circ} 652$ - HRC $245=\mathrm{HM} 13-\mathrm{Sz} 19-\mathrm{T} 26$ - IRAS 11059-7721. The visually brightest late-type star in the cloud $(V \propto 10.7)$, this is a G2 III star with weak emission lines (Ha only, with E.W. - $17 \mathrm{~A}$ ) and little photometric variability. It has a close visual companion to the south. Possible optical polarization variability has been reported in the blue, and it shows a rotation rate of $30 \pm$ $5 \mathrm{~km} \mathrm{~s} s^{-1}$. [Henize and Mendoza 1973; Grasdalen et al. 1975; Rydgren 1980; Bastien 1985; Bouvier et al. 1986]

CHX 10. No previously catalogued star appears in this X-ray error circle. Our spectra of the most prominent candidate shows a probable new cloud member. It is a reddened $\mathrm{V}=13.5 \mathrm{k} 7 \mathrm{star}$ with no emission lines. [This paper, Table 3]

CHX 11. VW Cha $=$ HM $17-\mathrm{S} 6343-\mathrm{Sz} 24-\mathrm{T} 31$. A highly variable T Tauri star $(14.5<\mathrm{V}<12.5)$, with strong Balmer lines, strong helium lines, and both P Cygni and inverse-P Cygni line profiles. Photometrically it has both a strong ultraviolet and infrared excess, and possible polarization variability. The spectral type is tentatively reported to be G1. [Henize and Mendoza 1973; Appenzeller 1977; Rydgren 1980; Bastien 1985] 
$\mathrm{Sz} 23=\mathrm{T} 30$. A second catalogued star in the error circle of $\operatorname{CHX} 11$, this is a faint $(R \simeq 16)$, weak-emission line star. No detailed properties are known. [Schwartz 1977]

CHX 12. Glass I = T 33 - IRAS 11068-7717. This star was not found in any Ho survey during the 1950-70's, and exhibited constant brightness $(V-12.83 \pm 0.06)$ on several plates obtained during the mid-1980's. It was originally considered to be a field star. However, it shows a strong infrared excess and $\mathrm{Ca}$ II $\mathrm{H}$ and $\mathrm{K}$ emission cores, leading whittet et al. to consider it a cloud member. Our CTIO observations show a close double consisting of a brighter K3 star with emission lines and a fainter G5 star with a weak $\mathrm{H} \alpha$ emission line. The latter, at least, is likely to be a cloud member, though it is not clear which is responsible for the X-ray emission. [Glass 1979; Whittet et al. 1987; this paper, Table 3]

CHX 13. A previously unidentified location, our spectra of two stars in the IPC error circle reveal a likely cloud member. It is a highly reddened $V=15.7$ M0 star with a weak Ha line. [This paper, Table 3]

$\mathrm{CHX}$ 14. Sz $30=\mathrm{T} 39=\mathrm{C} 7-7$. A moderately bright $(\mathrm{R} \approx 13)$ emission line star with relatively weak $\mathrm{H} \alpha$ and $\mathrm{Ca}$ II emission lines, and no infrared excess. It was missed in early epoch objective prism surveys. No detailed properties are known. [Schwartz 1977; Hyland et al. 1982]

CHX 15. WY Cha = HM 26 - S 6351 - Sz 36 - T 46 - IRAS 11085-7613. A T Tauri star with moderate intensity Balmer lines, strong lithium absorption and an infrared excess. Its spectral type is reported to be 
KO: and K7. [Henize and Mendoza 1973; Glass 1979; Rydgren 1980] Sz $33-\mathrm{T} 43=\mathrm{C} 1-17$. A second star in the $\mathrm{X}$-ray error circle, this was reported to be $a \mathrm{~V} \approx 15$ emission line star with weak $\mathrm{H} \alpha$ and no infrared excess. It was missed in early epoch surveys. Our spectrum shows it to be a V $=14.0 \mathrm{Ml}$ star with weak Ha lying very far above the ZAMS. [Schwartz 1977; Jones et al. 1985; this paper, Table 3]

CHX 16. WW Cha $=$ HM $24=\mathrm{S} 6348=\mathrm{Sz} 34=\mathrm{T} 44=\mathrm{C} 1-7$. A highly variable $(16.3<\mathrm{V}<13.5)$ T Tauri star with moderate to weak $\mathrm{H \alpha}$, a veiled spectrum and strong infrared excess. The spectral type is uncertain due to the veiling, but is estimated to be G - K5. [Henize and Mendoza 1973; Glass 1979; Rydgren 1980]

$\operatorname{CHX} 17$. WX Cha $=$ HM $25=\mathrm{S} 6349=\mathrm{Sz} 35-\mathrm{T} 45=\mathrm{C} 8-3$ - IRAS 11085-7720. A V $\approx 14 \mathrm{~T}$ Tauri star with moderate to strong Ho emission and K7-MO spectral type. [Henize and Mendoza 1973; Schwartz 1977; Rydgren 1980]

CHX 18. XX Cha $=$ HM 29 - Sz 39 - T 49 - IRAS 11101-7603. A relatively faint MO T Tauri star with high photometric variability $(16.4<\mathrm{V}$ < 13.4), strong and variable Balmer lines, inverse P Cygni profiles, strong ultraviolet and infrared excesses. [Henize and Mendoza 1973; Grasdalen et al. 1975; Appenzeller 1979; Rydgren 1980]

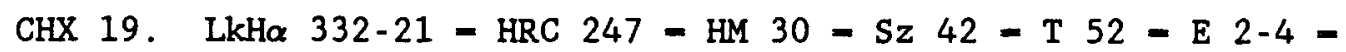
IRAS 11108-7627. A very bright and photometrically constant $(V=10.9)$ T Tauri star with spectral type G8, strong and probably variable Balmer lines, Ca II emission lines, strong lithium absorption, and infrared 
excess. [Henize and Mendoza 1973; Schwartz 1977; Glass 1979; Rydgren 1980]

HM $31=\mathrm{Sz} 43-\mathrm{T}$ 53. A second $\mathrm{T}$ Tauri star in the $\mathrm{X}$-ray error circle, fainter $(V \simeq 14-15)$ and variable with moderate or strong Ha emission. Its spectral type is estimated to be $G$. [Henize and Mendoza 1973; Rydgren 1980]

CHX 20. Sz $41=\mathrm{T} 51$ - E 1-9a = IRAS 11108-7620. A bright $(\mathrm{R} \simeq$ 11) star with very weak $\mathrm{H} \alpha$ emission, originally discovered by virtue of its $\mathrm{Ca}$ II $\mathrm{H}$ and $\mathrm{K}$ emission cores. Our spectrum shows it is an unreddened K3 star with V = 11.7. [Schwartz 1977; this paper, Table 3]

CHX 21. No previously catalogued star appears in this error circle. Our spectrum shows a likely new cloud member, an unreddened V = 13.3 K7 star with no detectable emission lines. [This paper, Table 3]

CHX 22. HM 'Anon' - T 54 - IRAS 11111-7705. A V = 11.1 star illuminating a small reflection nebula. It is photometrically very constant and shows no emission lines at all. The spectral type is G5 - KO. [Henize and Mendoza 1973; Rydgren 1980; Schwartz and Henize 1983; this paper, Table 3] 
Table 1

Log of X-ray Observations

$\begin{array}{ccccc}\text { IPC } & \text { Field Center } & \text { Roll } & \text { Exposure } \\ \text { Seq. No. } & \text { R.A. } & \text { Dec. } & \text { (P.A.) } & \text { (sec) } \\ & \text { h } m & \text { o }, & 0 & \end{array}$

$\begin{array}{lllllll}10349 & 11 & 0 & -77 & 10 & -143 & 2191 \\ 10359 & 11 & 7 & -77 & 30 & -147 & 3128 \\ 10351 & 11 & 8 & -76 & 30 & -141 & 2278\end{array}$


Table 2

Chamaeleon X-ray Sources and Suggested Optical Counterparts

$\mathrm{X}$-ray Data

Optical Candidates

\begin{tabular}{|c|c|c|c|c|c|c|c|c|c|c|c|c|c|c|}
\hline $\mathrm{CHX}$ & $\mathrm{h}$ & $\underset{\mathrm{m}}{\mathrm{R} . \mathrm{f}}$ & A. ${ }_{\mathrm{s}}^{(19}$ & 50) & Dec: & $\begin{array}{l}\text { Error } \\
\text {, }\end{array}$ & Counts & $\mathrm{s} / \mathrm{N}$ & Flux & Notes & $T$ & Name & $\Delta \theta$ & CTIO \\
\hline 1 & 10 & 55 & 04.0 & -76 & 55.6 & 1.1 & $20.4 \pm 5.2$ & 3.8 & 4.9 & & 4 & SY Cha & 0.8 & \\
\hline 2 & 10 & 56 & 57.6 & -77 & 01.6 & 1.0 & $18.4 \pm 5.1$ & 3.6 & 3.3 & & 6 & sz Cha & 0.6 & \\
\hline 3 & 10 & 57 & 49.8 & -76 & 45.7 & 1.0 & $73.6 \pm 9.0$ & 8.2 & 19.1 & & 8 & HM 4 & 0.2 & \\
\hline 4 & $\begin{array}{l}11 \\
11\end{array}$ & $\begin{array}{l}00 \\
01\end{array}$ & $\begin{array}{l}59.9 \\
06.7\end{array}$ & $\begin{array}{l}-77 \\
-77\end{array}$ & $\begin{array}{l}17.5 \\
17.0\end{array}$ & $\begin{array}{l}0.6 \\
1.0\end{array}$ & $\begin{array}{l}33.2 \pm 6.3 \\
52.4 \pm 7.9\end{array}$ & $\begin{array}{l}5.3 \\
6.8\end{array}$ & $\begin{array}{l}5.8 \\
8.8\end{array}$ & & 11 & $\mathrm{HM} 7$ & 0.4 & \\
\hline 5 & 11 & 01 & 48.8 & -77 & 58.8 & 0.9 & $13.3 \pm 4.4$ & 3.1 & 3.6 & & $\ldots$ & & & \\
\hline 6 & 11 & 04 & 23.1 & -76 & 51.2 & 0.8 & $13.7 \pm 4.4$ & 3.1 & 3.4 & & $\ldots$ & $\begin{array}{l}\text { Cand } a \\
\text { Cand } b\end{array}$ & & $\begin{array}{l}\mathrm{x} \\
\mathrm{x}\end{array}$ \\
\hline 7 & $\begin{array}{l}11 \\
11\end{array}$ & $\begin{array}{l}04 \\
04\end{array}$ & $\begin{array}{l}52.6 \\
47.6\end{array}$ & $\begin{array}{l}-77 \\
-77\end{array}$ & $\begin{array}{l}05.5 \\
05.8\end{array}$ & $\begin{array}{l}0.5 \\
1.0\end{array}$ & $\begin{array}{l}70.9 \pm 8.9 \\
96.5 \pm 10.2\end{array}$ & $\begin{array}{l}8.0 \\
9.4\end{array}$ & $\begin{array}{l}13.3 \\
18.6\end{array}$ & & 21 & Ced 110 & 0.3 & $x$ \\
\hline 8 & 11 & 05 & 19.1 & -77 & 11.2 & 1.1 & $15.6 \pm 4.8$ & 3.3 & 3.2 & & 22 & UX Cha & 0.7 & \\
\hline 9 & 11 & 06 & 02.4 & -77 & 22.0 & 0.7 & $21.4 \pm 5.5$ & 3.9 & 2.5 & (1) & 26 & HM 13 & 0.3 & \\
\hline 10 & $\begin{array}{l}11 \\
11\end{array}$ & $\begin{array}{l}06 \\
06\end{array}$ & $\begin{array}{l}41.4 \\
25.7\end{array}$ & $\begin{array}{l}-77 \\
-77\end{array}$ & $\begin{array}{l}11.7 \\
11.0\end{array}$ & $\begin{array}{l}0.6 \\
1.1\end{array}$ & $\begin{array}{l}18.4 \pm 4.8 \\
12.9 \pm 4.3\end{array}$ & $\begin{array}{l}3.9 \\
3.1\end{array}$ & $\begin{array}{l}2.7 \\
3.1\end{array}$ & (2) & $\ldots$ & Cand a & & $\mathrm{x}$ \\
\hline 11 & $\begin{array}{l}11 \\
11\end{array}$ & $\begin{array}{l}06 \\
06\end{array}$ & $\begin{array}{l}44.5 \\
45.0\end{array}$ & $\begin{array}{l}-77 \\
-77\end{array}$ & $\begin{array}{l}26.0 \\
26.0\end{array}$ & $\begin{array}{l}0.6 \\
1.0\end{array}$ & $\begin{array}{l}34.5 \pm 6.7 \\
25.0 \pm 5.6\end{array}$ & $\begin{array}{l}5.3 \\
4.5\end{array}$ & $\begin{array}{l}3.8 \\
7.2\end{array}$ & (3) & $\begin{array}{l}31 \\
30\end{array}$ & $\begin{array}{l}\text { VW Cha } \\
\text { Sz } 23\end{array}$ & $\begin{array}{l}0.4 \\
0.7\end{array}$ & \\
\hline 12 & 11 & 06 & 53.0 & -77 & 18.1 & 0.7 & $27.1 \pm 6.0$ & 4.3 & 3.3 & & 33 & Glass I & 0.6 & $\mathrm{x}$ \\
\hline 13 & 11 & 07 & 24.2 & -76 & 19.7 & 1.0 & . & -3 & 2.2 & (4) & $\cdots$ & $\begin{array}{l}\text { Cand a } \\
\text { Cand b } \\
\mathrm{C} 1-10\end{array}$ & 1.0 & $\begin{array}{l}\mathrm{x} \\
\mathrm{x}\end{array}$ \\
\hline
\end{tabular}




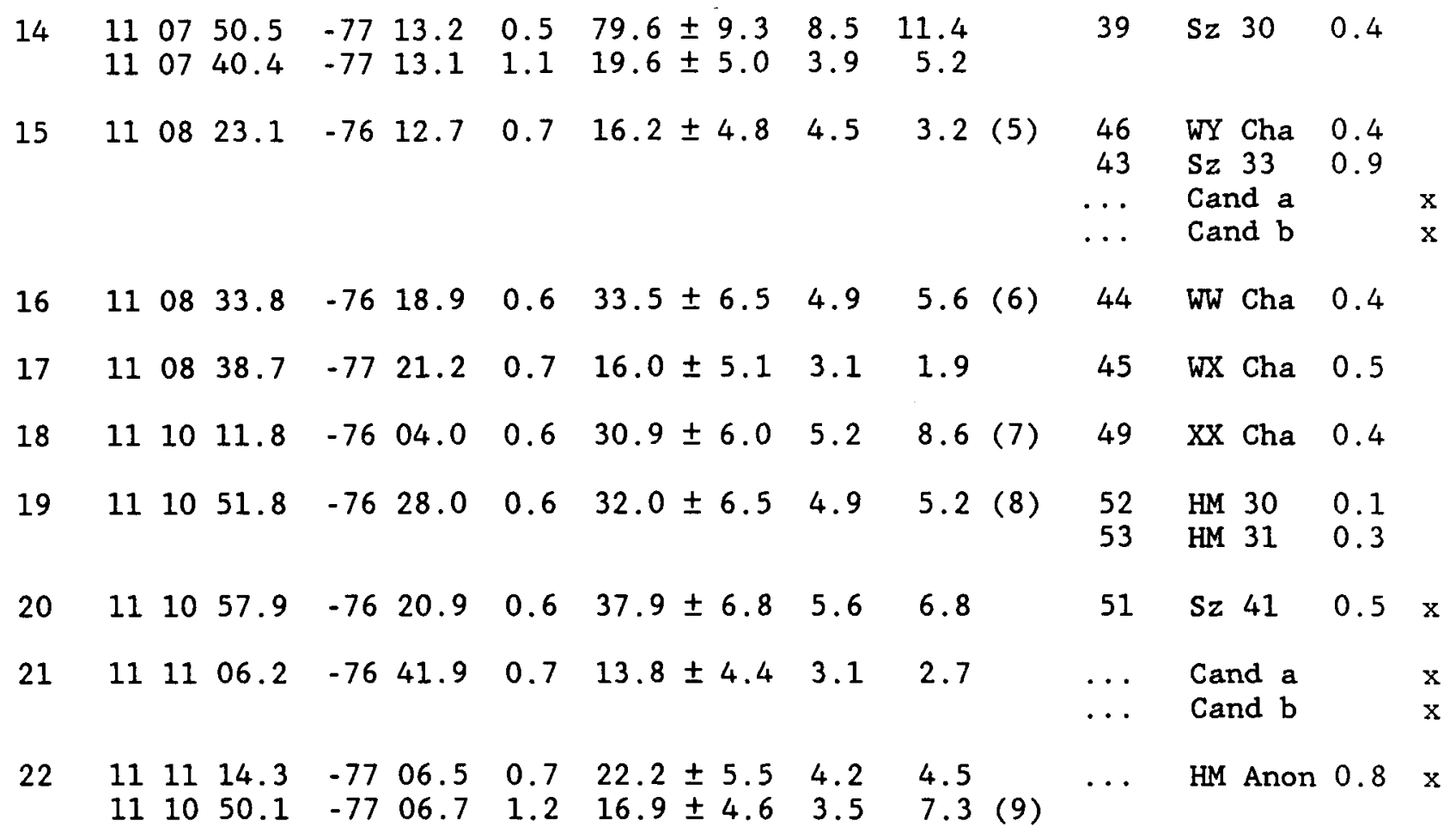

Notes to Table 2:

(1) Elongated E-W on IPC contour map, possibly due to a secondary source 2' $E$ of the stated centroid. This may be due to HM $16=\mathrm{Sz} 22$.

(2) IPC window support structure to the NW. True IPC position probably NW of stated position.

(3) Elongated N-S on IPC contour map, possibly due to a secondary source 1' $S$ of stated centroid.

(4) Detected as a significant source only in the Rev. 0 processing of image 10351. Stated flux is at the Rev. 1B threshold.

(5) Elongated E-W on IPC contour map, possibly due to a secondary source 2' WNW of stated centroid.

(6) Elongated $\mathrm{N}-\mathrm{S}$ on IPC contour map, possibly due to two sources $\sim 0.7^{\prime}$ $\mathrm{N}$ and $\mathrm{S}$ of stated centroid. The northern source might be $\mathrm{HM} 23-\mathrm{Sz} 32$.

(7) Window support structures to the NW and SW. True IPC position may be NW, $W$ or SW of stated position.

(8) Suggestion of elongated structure in IPC contour map, possibly due to a second source $1.5^{\prime} \mathrm{N}$ of the stated centroid.

(9) Window support structure to the SE, edge of field to the NE. True IPC position may be $\mathrm{NE}, \mathrm{E}$, or $\mathrm{SE}$ of stated position. 
Table 3

CTIO Observations of New Optical Candidates

\begin{tabular}{|c|c|c|c|c|c|c|c|c|}
\hline $\mathrm{CHX}$ & Sp Ty & $\mathbf{R}$ & $V-R$ & $B-V$ & $E(B-V)$ & $\begin{array}{c}F(\mathrm{H \alpha}) \\
10(-14)\end{array}$ & $\begin{array}{c}\mathrm{EW}(\mathrm{H} \alpha) \\
(\mathrm{A})\end{array}$ & Notes \\
\hline $\begin{array}{r}6 a \\
b \\
7 \\
10 \\
12 a \\
b \\
13 a \\
b \\
15 a \\
b \\
20 a \\
b \\
21 a \\
b \\
22\end{array}$ & $\begin{array}{l}\text { K7 } \\
\text { MO } \\
\text { G5+2 } \\
\text { K7 } \\
\text { K3 } \\
\text { G5e+2 } \\
\text { MOe } \\
\text { G4+4 } \\
\text { A7 } \\
\text { M1e } \\
\text { K0+2 } \\
\text { K3e } \\
\text { K7 } \\
\text { F3 } \\
\text { G8+2 }\end{array}$ & $\begin{array}{r}11.99 \\
11.94 \\
9.78 \\
11.78 \\
11.90 \\
12.52 \\
13.85 \\
14.98 \\
9.68 \\
12.37 \\
11.63 \\
10.67 \\
12.06 \\
13.87 \\
10.28\end{array}$ & $\begin{array}{l}1.15 \\
1.98 \\
1.49 \\
1.72 \\
1.38 \\
1.57 \\
1.84 \\
1.12 \\
0.84 \\
1.66 \\
1.10 \\
1.00 \\
1.28 \\
1.09 \\
0.98\end{array}$ & $\begin{array}{l}1.34 \\
2.25 \\
1.53 \\
1.88 \\
1.35 \\
1.63 \\
2.09 \\
1.30 \\
0.81 \\
1.79 \\
1.36 \\
1.13 \\
1.49 \\
1.07 \\
1.13\end{array}$ & $\begin{array}{r}-0.04 \pm 0.12 \\
0.80 \pm 0.12 \\
0.83 \pm 0.13 \\
0.50 \pm 0.12 \\
0.25 \pm 0.12 \\
0.93 \pm 0.13 \\
0.64 \pm 0.12 \\
0.62 \pm 0.16 \\
0.62 \pm 0.11 \\
0.30 \pm 0.12 \\
0.47 \pm 0.13 \\
0.03 \pm 0.12 \\
0.11 \pm 0.12 \\
0.71 \pm 0.11 \\
0.39 \pm 0.14\end{array}$ & $\begin{array}{c}<3.2 \\
<1.7 \\
<10.0 \\
<1.8 \\
\cdots .7 \\
39 . \pm \pm 4 . \\
1.9 \pm 0.1 \\
-0.7 \pm 0.1 \\
-170 \pm 13 \\
9.5 \pm 0.3 \\
-8.4 \pm 2.6 \\
26 . \pm 0.4 \\
<1.9 \\
-1.5 \pm 0.3 \\
-20 . \quad \pm 7 .\end{array}$ & $\begin{array}{c}<2.4 \\
<1.6 \\
<1.2 \\
<1.3 \\
\cdots \pm \\
9.3 \pm 1.0 \\
4.5 \pm 0.3 \\
-4.5 \pm 0.7 \\
-7.4 \pm 0.6 \\
6.1 \pm 0.4 \\
-2.3 \pm 0.7 \\
3.0 \pm 0.5 \\
<1.6 \\
-3.4 \pm 0.6 \\
-1.5 \pm 0.5\end{array}$ & $\begin{array}{l}(3) \\
(4) \\
(5)\end{array}$ \\
\hline 3) & $\begin{aligned} \text { - Ced } \\
\text { - Glas } \\
\text { of t } \\
\text { star } \\
\text { estir } \\
\text { - Sz } 3 \\
\text { - Sz } 4 \\
\text { - HM A }\end{aligned}$ & $\begin{array}{l}0 . \\
\text { I. } \\
\text { com } \\
\text { whil } \\
\text { ted. } \\
\text { n. }\end{array}$ & $\begin{array}{l}\text { e Ap } \\
\text { is s } \\
\text { nent } \\
\text { the }\end{array}$ & $\begin{array}{l}\text { dix } \\
\text { is } \\
\text { The } \\
\text { stre }\end{array}$ & $\begin{array}{l}\text { or other nam } \\
\text { close doubl } \\
\text { Ho emission } \\
\text { gth of the b }\end{array}$ & $\begin{array}{l}\text { and infor } \\
\text { The } R \text { mag } \\
\text { iginates } \\
\text { ghter can }\end{array}$ & $\begin{array}{l}\text { ion on thes } \\
\text { ude refers } \\
\text { in the fai } \\
\text { be reliabl }\end{array}$ & $\begin{array}{l}\text { e stars. } \\
\text { to the sum } \\
\text { nter } \\
\text { y }\end{array}$ \\
\hline
\end{tabular}


Table 4

X-ray and Optical Properties of Chamaeleon T Tauri Stars

\begin{tabular}{rrrrr}
$\mathrm{Sz}$ & $\mathrm{HM}$ & \multicolumn{1}{l}{$\log L_{\mathbf{x}}$} & \multicolumn{1}{c}{$m_{r}$} & $\mathrm{H} \alpha$ index \\
2 & 1 & $<29.86$ & 14.2 & 2 \\
3 & 2 & 30.08 & 12.5 & 2 \\
5 & 3 & $<29.81$ & 12.5 & 4 \\
6 & 4 & 30.65 & 10.9 & 3 \\
7 & 5 & $<30.08$ & 16.2 & 0 \\
& & & & \\
9 & 7 & 30.23 & 11.2 & 3 \\
10 & 8 & $<29.75$ & 15.0 & 3 \\
11 & 9 & $<29.98$ & 11.5 & 3 \\
12 & 10 & $<29.77$ & 14.8 & 0 \\
13 & $\ldots$ & $<29.83$ & 16.5 & 2 \\
& & & & \\
14 & $\ldots$ & $<29.67$ & 16.7 & 1 \\
15 & $\ldots$ & $<29.67$ & 13.6 & 1 \\
16 & 11 & $<29.89$ & 14.9 & 1 \\
19 & 13 & 29.77 & 10.0 & 1 \\
20 & 14 & $<29.73$ & 13.5 & 5 \\
& & & & \\
21 & 15 & $<29.63$ & 14.2 & 4 \\
29 & 21 & $<29.79$ & 13.8 & 4 \\
30 & $\ldots$ & 30.29 & 12.6 & 1 \\
34 & 24 & 30.12 & 12.1 & 3 \\
35 & 25 & 29.65 & 13.8 & 3 \\
37 & 27 & $<29.91$ & 15.7 & 3 \\
38 & 28 & $<29.75$ & 15.2 & 2 \\
39 & 29 & 30.30 & 14.2 & 5 \\
40 & $\ldots$ & $<29.75$ & 15.2 & 2 \\
41 & $\ldots$ & 30.20 & 11.2 & 1 \\
& & & & \\
44 & $\ldots$ & $<29.82$ & 15.4 & 1 \\
& & & &
\end{tabular}




\section{References}

Allen, C. W. 1973, Astrophysical Quantities, London:Athlone.

Appenzeller, I. 1977, Astron. Astrophys. 61, 21.

Appenzeller, I. 1979, Astron. Astrophys. 71, 305.

Appenzeller, I., Jankovics, I., and Krautter, J. 1983, Astron. Astrophys. Supp1. 53, 291.

Avni, Y., Soltan, A., Tananbaum, H., and Zamorani, G. 1980, Ap. J. 238, 800.

Bastien, P. 1985, Ap. J. Supp1. 59, 277.

Baud, B. et al. 1984, Ap. J. Lett. 278, L53.

Bertout, C. 1984, Rep. Prog. Phys. 47, 111.

Bouvier, J., Bertout, C., Benz, W., and Mayor, M. 1986. Astron. Astrophys. 165, 110.

Cohen M. 1984, Phys. Rept. 116, 173.

Cohen, M. and Kuhi, L. V. 1979, Ap. J. Supp1. 41, 743.

Feigelson, E. D. 1984, in Cool Stars, Stellar Systems and the Sun (S. L. Baluinas and L. Hartmann, eds.) Berlin:Springer, p. 27.

Feigelson, E. D. 1987, in Protostars and Molecular Clouds (T. Montmerle and C. Bertout, eds.) Saclay:CEA, p. 123.

Feigelson, E. D. and DeCampli, W. M. 1981, Ap. J. Lett. 243, L89.

Feigelson, E. D. and Kriss, G. A. 1981, Ap. J. Lett. 248, L35.

Feigelson, E. D. and Nelson, P. I. 1985, Ap. J. 293, 192.

Feigelson, E. D., Jackson, J. M., Mathieu, R. D., Myers, P. C. and Walter F. M. 1987, Astr. J. 94, 1251.

Giacconi, R. et al. 1979, Ap. J. 230, 540.

Glass, I. S. 1979, M.N.R.A.S. 187, 305.

Grasdalen, G., Joyce, R., Knacke, R. F., Strom, S. E. and Strom, K. M. 1975, Astron. J. 80, 117. 
Heck, A. and Warren, W. H. 1986, B.A.A.S. 18, 817.

Henize, K. G. and Mendoza, E. E. 1973, Ap. J. 180, 115.

Herbig. G. and Rao, N. K. 1972, Ap. J. 174, 401.

Hoffmeister, C. 1963, Veroff. Sternwarte Sonneberg, Vol. 6, No. 1.

Hyland, A. R., Jones, T. J. and Mitche11, R. M. 1982, M.N.R.A.S. 201, 1095.

Isobe, T., Feigelson, E. D. and Nelson, P. I. 1986, Ap. J. 306, 490.

Jones, T. J., Hyland, A. R., Harvey, P. M., Wilking, B. A., and Joy, M. 1985, Astron. J. 90, 1191.

Ku, W. H.-M., Righini-Cohen, G. and Simon, M. 1982, Science 215, 61.

Micela, G., Sciortino, S., Vaiana, G. S., Schmitt, J. H., Stern, R. A., Harnden, F. R. and Rosner, R. 1988, Ap. J. in press.

Montmerle, T., Koch-Miramond, L., Falgarone, E., and Grindlay, J. 1983, Ap. J. $269,182$.

Mundt, R. and Bastien, U. 1980, Astr. Astrophys. Supp1. 39, 245.

Mundt, R., Walter, F. M., Feigelson, E. D., Finkenzeller, U., Herbig, G., and Ode11, A. P. 1983, Ap. J. 269, 182.

Pritchet, C. and van den Bergh, S. 1977, Ap. J. Suppl. 34, 101.

Rydgren, A. E. 1980, Astron. J. 85, 444.

Schaeffer, B. E. 1983, Ap. J. Lett. 266, L45.

Schmitt, J. H. M. M., 1985, Ap. J. 293, 178.

Schwartz, R. D. 1977, Ap. J. Suppl. 35, 161.

Schwartz, R. D. and Henize, K. G. 1983, Astron. J. 88, 1165.

Shu, F. H., Adams, F. C. and Lizano, S. 1987, Ann. Rev. As. Ap. 25, 23.

Skumanich, A. 1972, Ap. J. 171, 565.

Toriseva, M., Hoglund, B. and Mattila, K. 1985, Rev. Mex. As. Astrof. 10, 135.

Toriseva, M. and Mattila, K. 1985, Astron. Astrophys. 153, 207.

Walter, F. M. 1986, Ap. J. 306, 573. 
Walter, F. M. 1987, P.A.S.P. 99, 31.

Walter, F. M. and Kuhi, L. V. 1981, Ap. J. 250, 254.

Walter, F. M. and Kuhi, L. V. 1984, Ap. J. 284, 194.

Walter, F. M. et al. 1987, Ap. J. 314, 297.

Walter, F. M., Brown, A., Mathieu, R. D., Myers, P. C., and Vrba, F. J. 1988 , in preparation.

Whittet, D. C. B., Kirrane, T. M., Kilkenny, D., Oates, A. P., Watson, F. G. and King, D. J. 1987, M.N.R.A.S. $224,497$.

Zoonematkermani, S. and Caillault, J. P. 1987, B.A.A.S. 19, 717. 
Figure 1. Composite contour map of the Chamaeleon IPC fields, after smoothing with a $1^{\prime}$ FWHM Gaussian.

Figure 2. Finding charts.

Figure 3. CTIO spectra.

Figure 4. Diagram of the Chamaeleon I cloud and its pre-main sequence stars. Crosses are the X-ray sources from Table 2 (this paper). Filled circles are the $\mathrm{H} \alpha$ emission line stars, squares are reflection nebulae, and H's are Herbig-Haro objects (Schwartz 1977). Hatches outline areas of heavy optical obscuration (approximately $A_{V}=4$, see Fig. 6 of Hyland et al. 1982).

Figure 5. Approximate Hertsprung-Russell diagram of some pre-main sequence stars in the Chamaeleon cloud, including optically selected strong $\mathrm{H} \alpha$ emitters (filled circles), X-ray selected weak $\mathrm{H} \alpha$ (crosses) and absent $\mathrm{H} \alpha$ (crosses with question marks) emitters.

Figure 6. Integral $\mathrm{X}$-ray luminosity functions for Chamaeleon (solid lines, this paper) and main sequence (dashed lines, taken from Micela et al. 1987) low mass stars, using the Kaplan-Meier estimator. (a) All X-ray sources in Table 2; (b) Ha emission line stars in Table 4; (c) Pleiades $\mathrm{K}$ stars; (d) Hyades K stars; (e) young disk K stars; and (f) old disk K stars. 
Figure 7. Plots of X-ray luminosity against (a) $R$ magnitudes and (b) index of Ha intensities from Schwartz (1977) for the sample of $\mathrm{H} \alpha$ emission stars given in Table 4. The correlation with $R$ is significant at the $0.05 \%$ confidence level according to a Cox regression test, and 0.38 confidence level according to a generalized Kendall's tau test. 


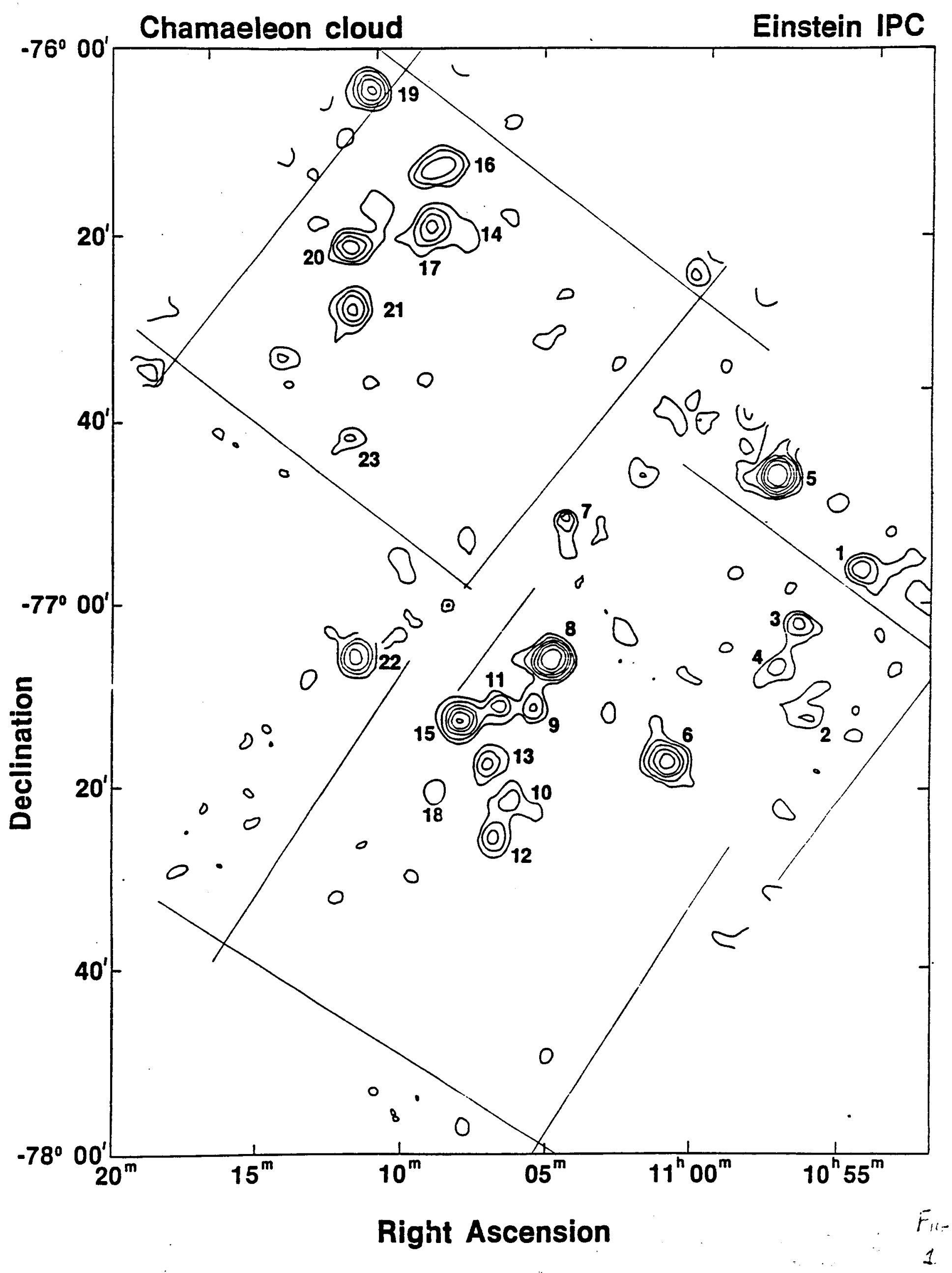



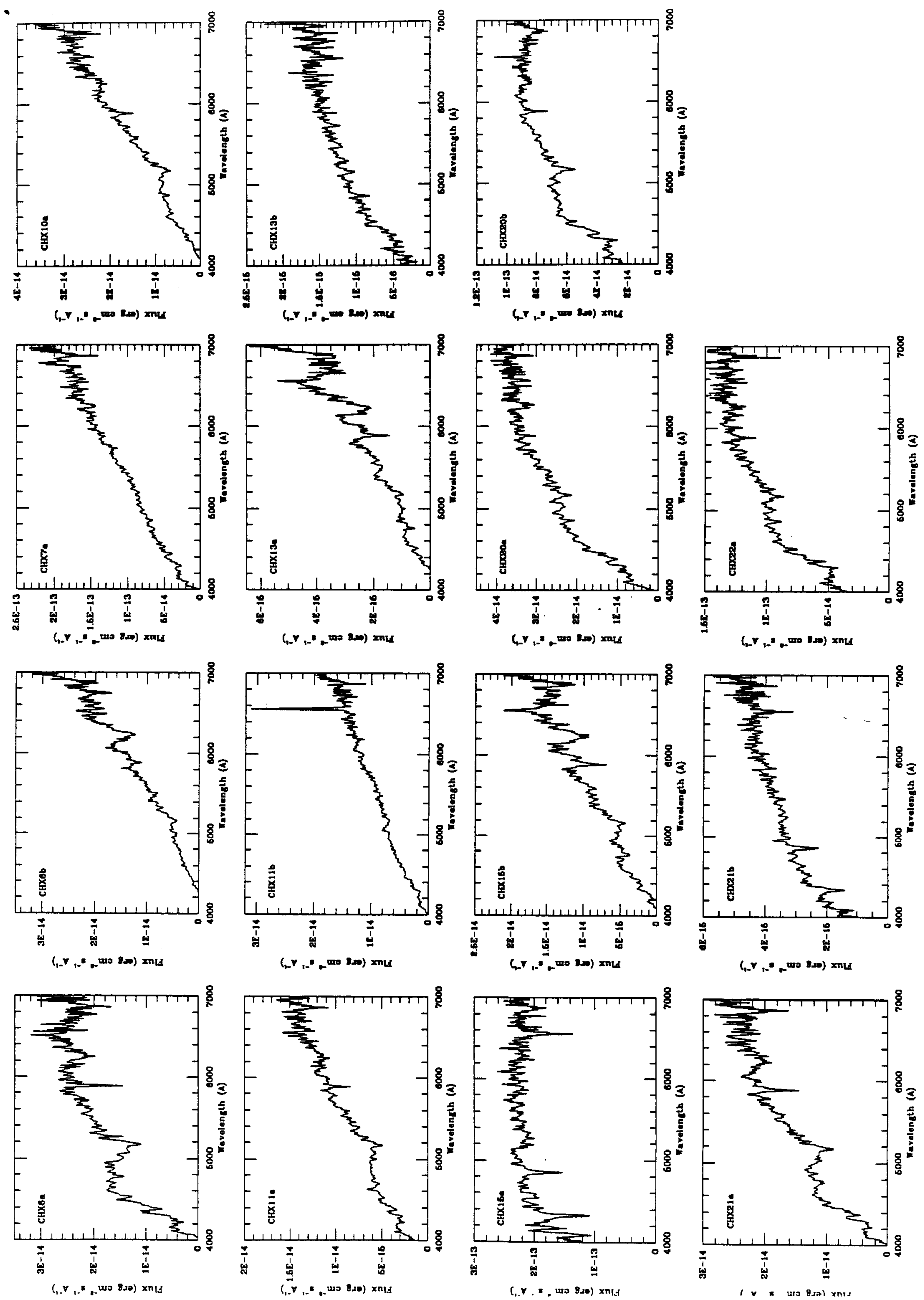


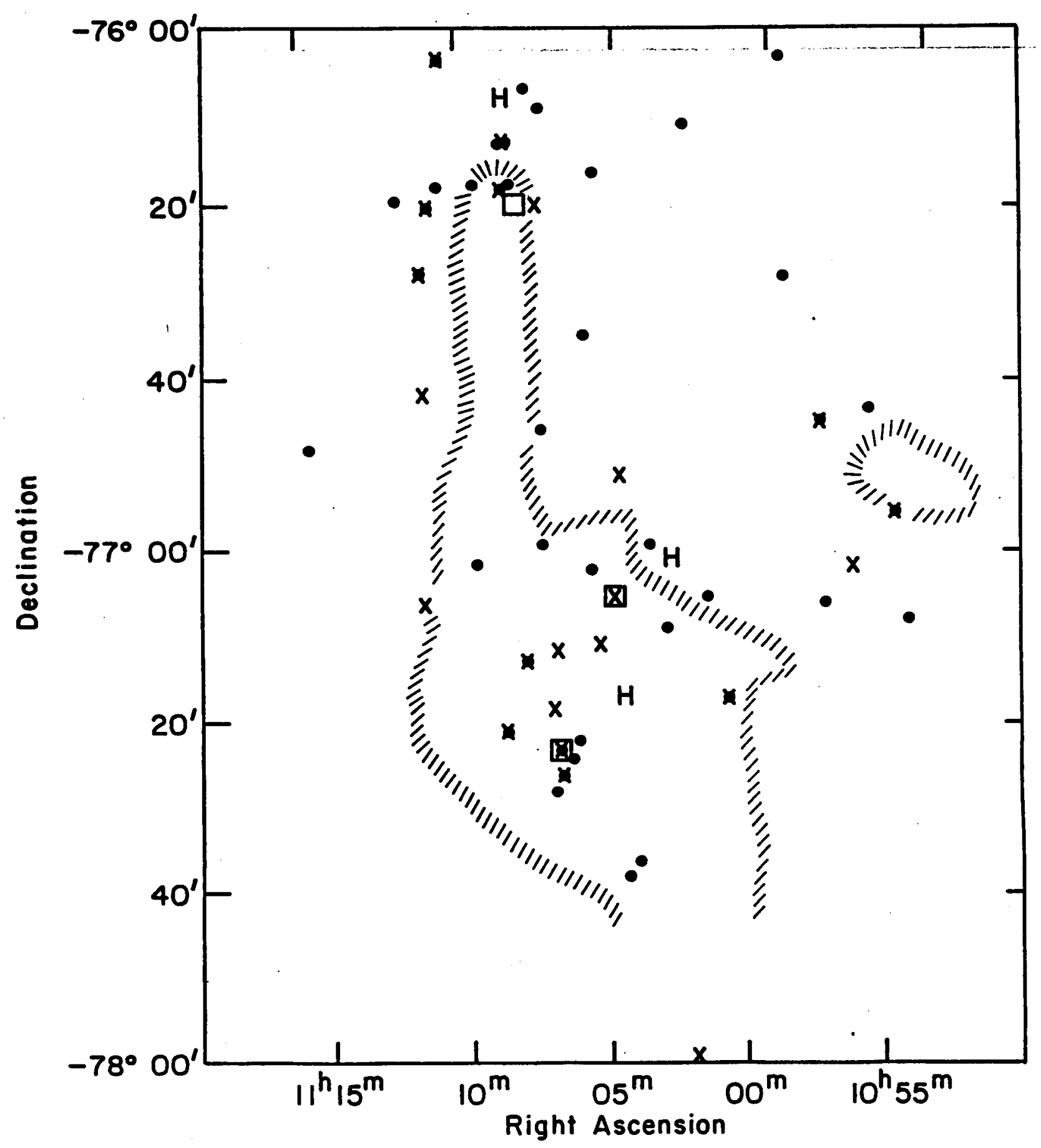




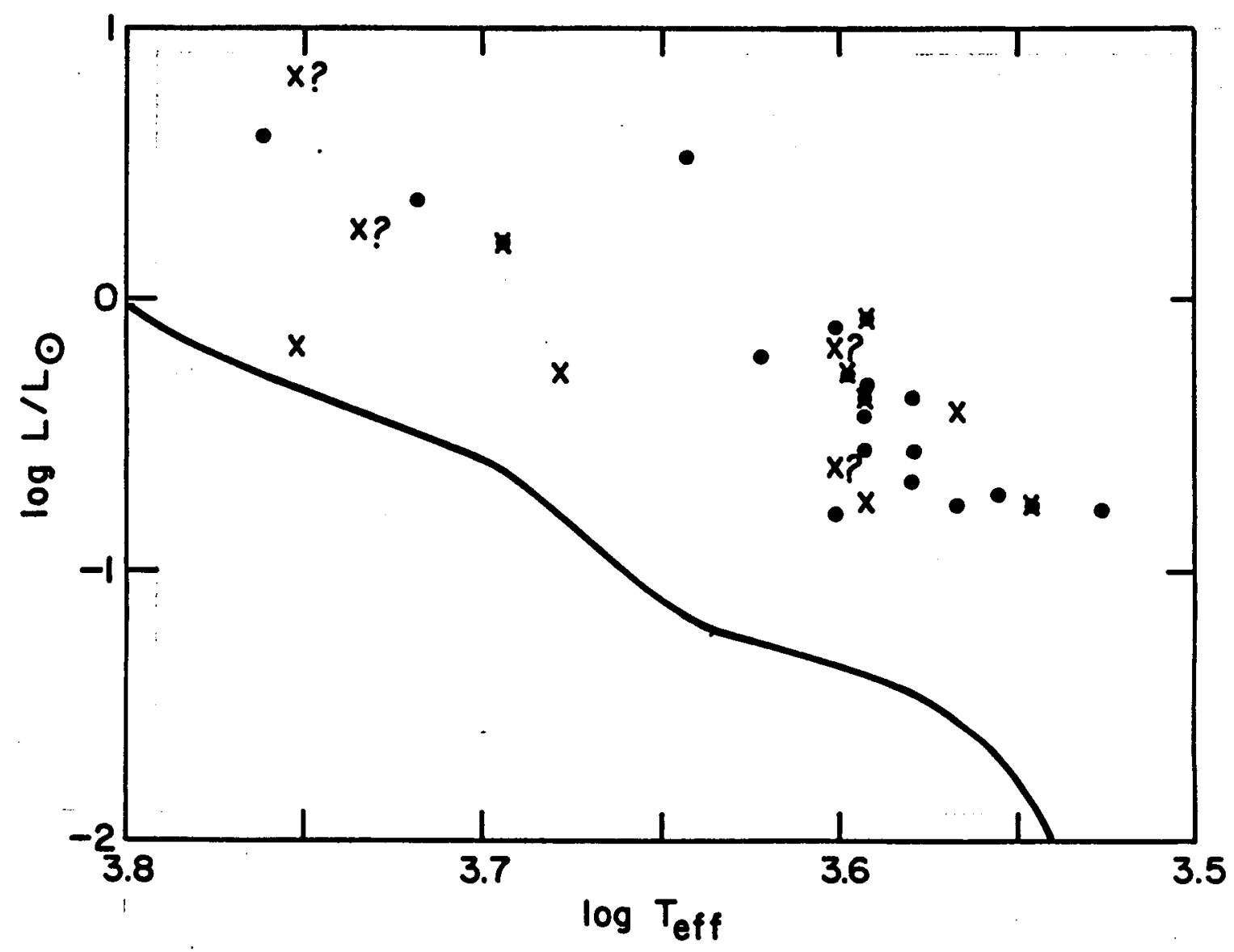




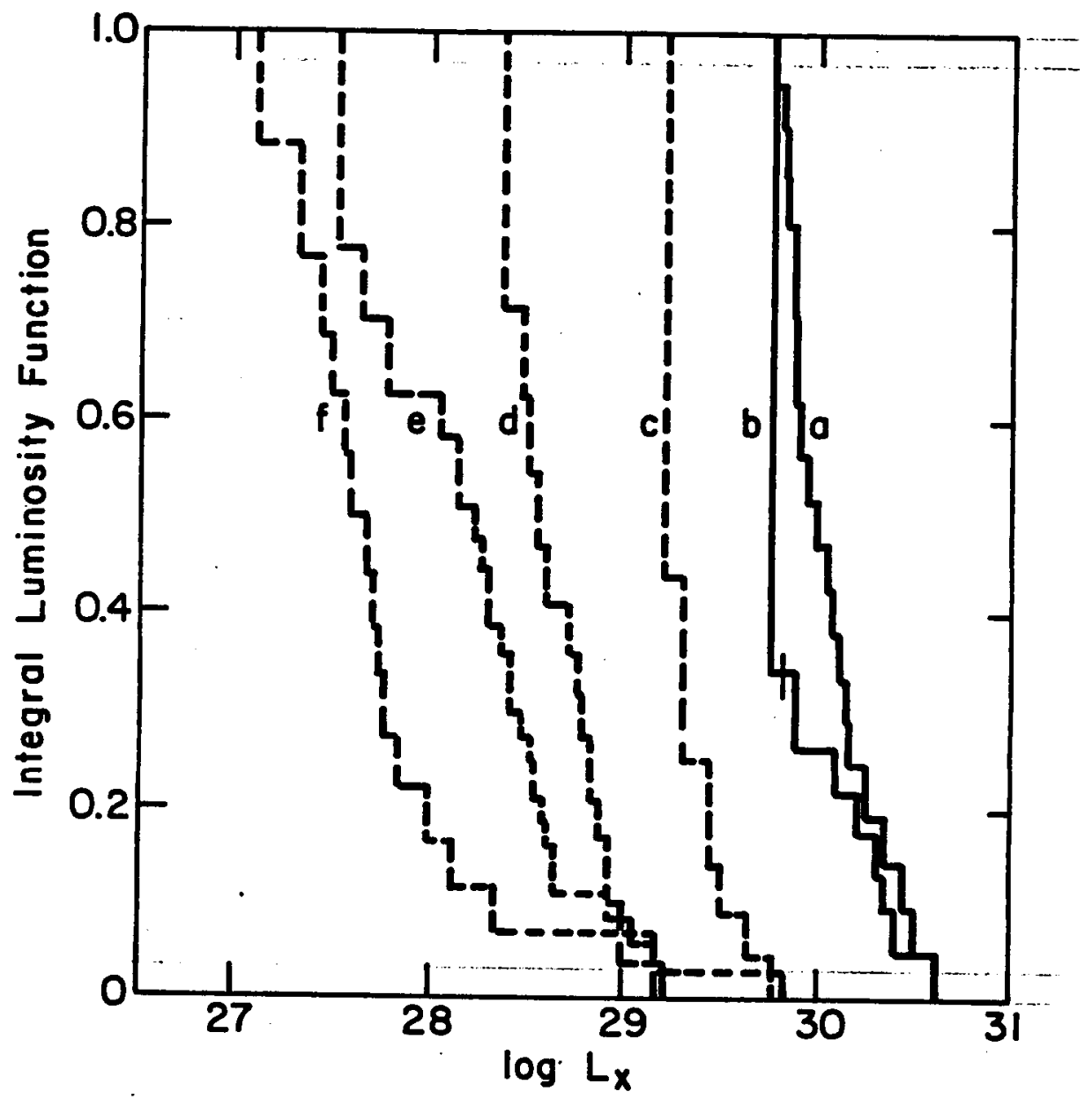



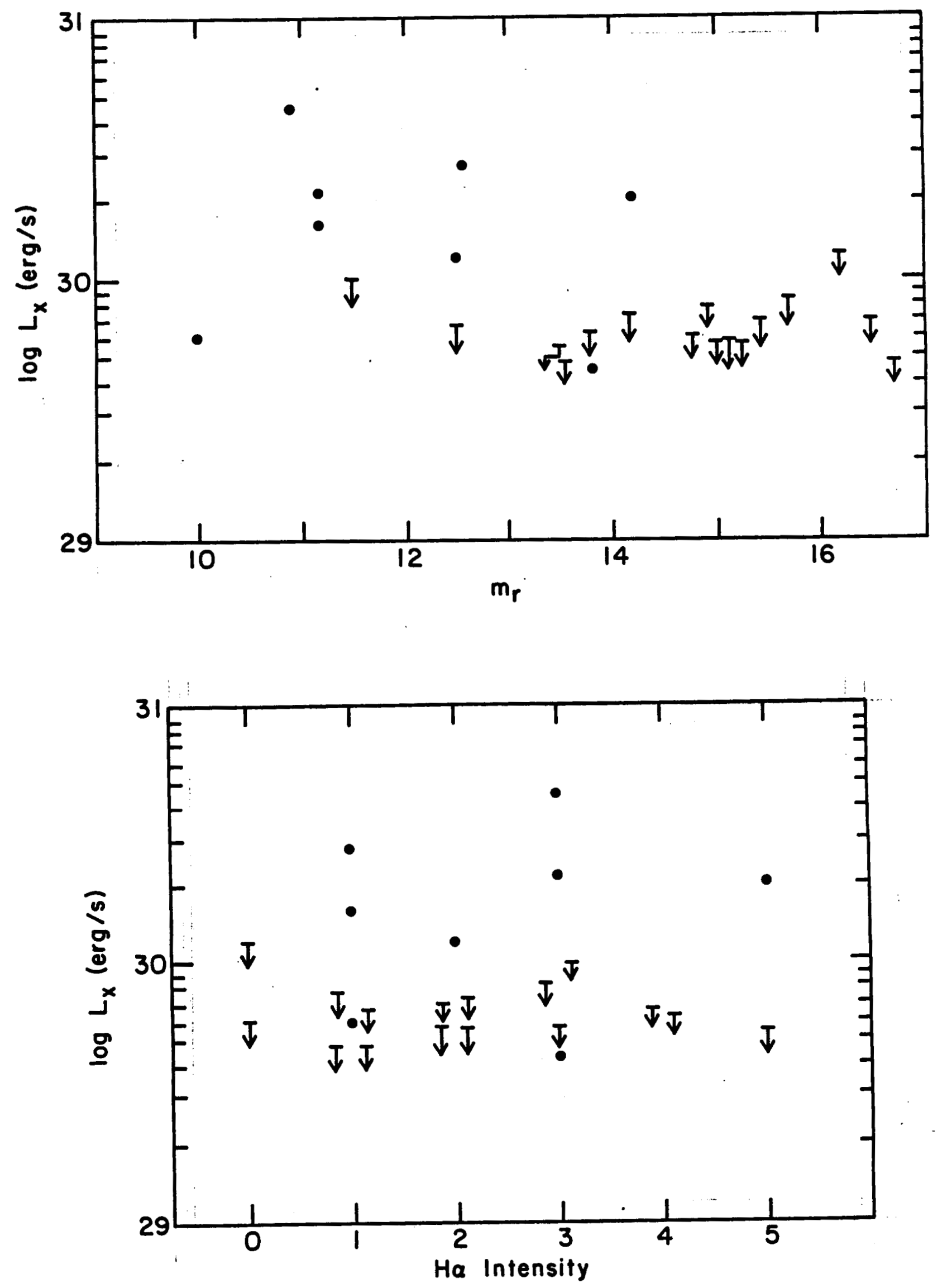


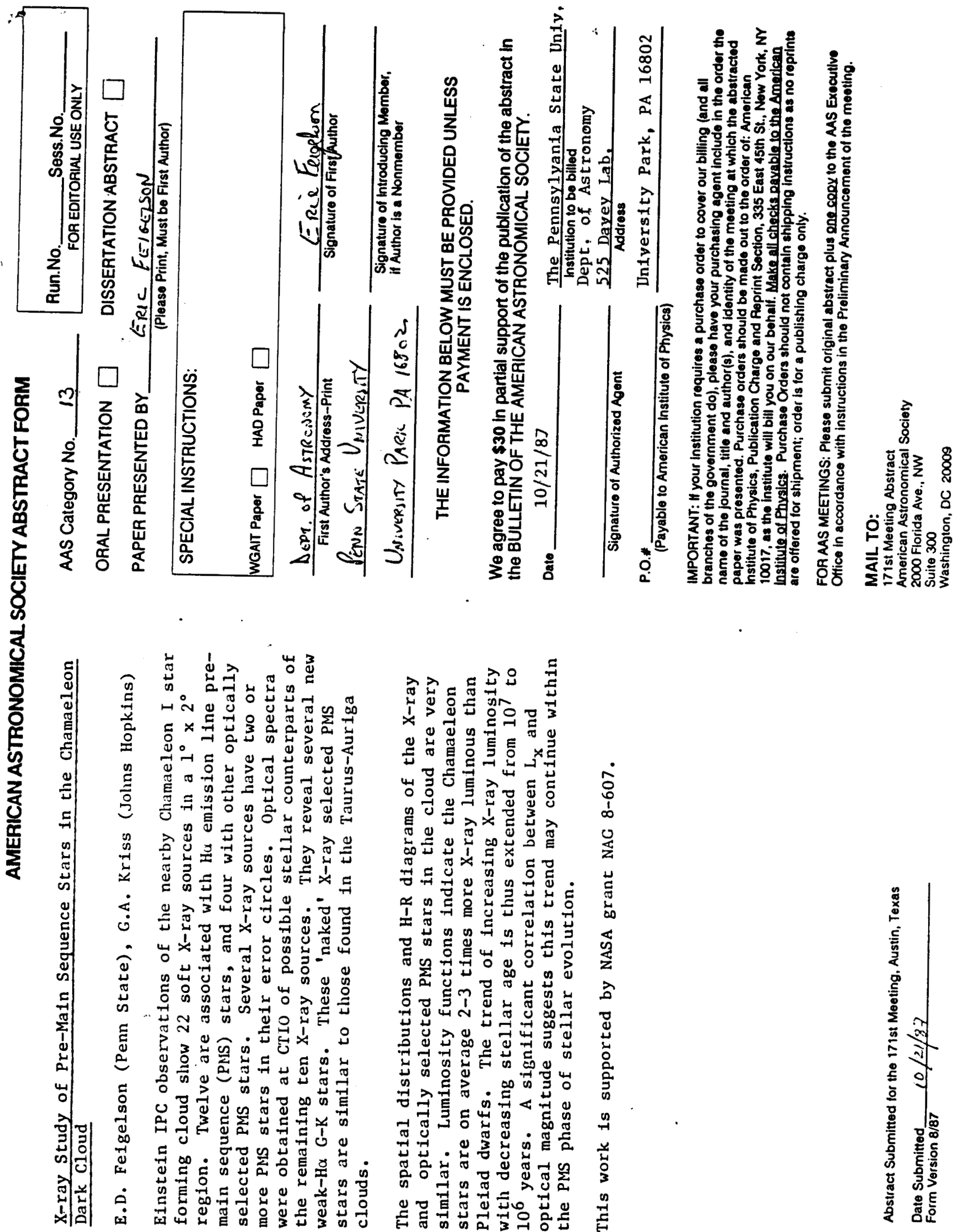

